\title{
In Silico Molecular Docking and Ab initio DFT Studies as a Tool for a Quick Screening on Drug Inhibitors for SARS-Cov-2 RNA-Dependent Polymerase
}

Manos C. Vlasiou ( $\nabla$ vlasiou.m@unic.ac.cy)

University of Nicosia https://orcid.org/0000-0002-8356-0868

Kyriakos I. loannou

University of Nicosia

Kyriaki S. Pafiti

University of Nicosia

\section{Research Article}

Keywords: Covid-19, Remdesivir, Molecular Docking, DFT Studies

Posted Date: August 2nd, 2020

DOl: https://doi.org/10.21203/rs.3.rs-50360/v1

License: (c) (1) This work is licensed under a Creative Commons Attribution 4.0 International License.

Read Full License 


\section{Abstract}

In silico molecular docking took place in order to evaluate already FDA approved drugs for several diseases, as inhibitors for SARSCov-2 RNA- dependent polymerase. The best candidates with the highest binding affinities, evaluated for their energy determined by their electron density. Remdesivir and Saquinavir seemed to be good candidates for clinical trials but their predicted toxicity based on their calculated structures revealed that these two substances should evaluated more for their side effects

\section{Introduction}

SARS-Cov-2 RNA- dependent polymerase is a viral protein that plays an important role for the severe acute respiratory syndrome coronavirus 2 [1-3]. The RNA-dependent RNA polymerase [(RdRp), also named nsp12] is the central component of coronaviral replication and transcription machinery, and it appears to be a primary target for the antiviral drug Remdesivir [4]. While traditional methods of drug discovery could take years, the approach taken to search possible medications for SARS-Cov-2 used in silico docking models for the spike glycoprotein and the sars-cov-2 3CL main protease reported elsewhere [5] with promising results. Recently, our research group reported the example of the interaction of already synthesized compounds with proved anticancer activity with several Covid-19 dependent proteins [6]. The development of a new vaccine, having in mind that recovering patients from Covid-19 disease didn't manage to create antibodies very soon in correlation with the fact that many countries are waiting for a new wave of Covid-19 pandemic, is driving us to a fact that the production of a drug that could face this disease is more urgent and feasible to be done [7,8]. The lack of time and the urgency of the situation turned the researchers to test on clinical trials drug molecules that already approved and used for other diseases. As far as now, several drug candidates disappointed the researchers and many clinical trials stopped. The data that we collected from drug molecules that either failed in clinical trials, or data of chemical structures that seems promising can be stored and evaluated by sophisticated software programs and make a good estimation of the drug that has to be created and give to the synthetic chemists valuable information before entering the laboratory. Herein, we are representing the computational evaluation use via molecular docking studies $[9,10]$ of 14 FDA approved drugs for various diseases specifically on nsp 12 protein. This is the strategy that researchers from all over the world applied, testing already approved drugs on Covid-19 clinical trials. We did this theoretically, with much less time and for the first time in our knowledge we make the use of DFT studies on the best candidates in order to find similarities on structures of that molecules that makes them good inhibitor candidates for nsp12. This knowledge, in correlation with the theoretical structural evaluation gave as valuable information about the toxicity of those candidates, a fact that should be studied further.

\section{Methods}

- Molecular Docking 
Molecular docking studies were carried out by using iGEMDOCK 2.1 software [11]. 6M71 coded crystal structure was selected from Protein Data Bank (www.rcsb.org). Ligand molecules were collected by Drug Bank (www.drugbank.ca). The scoring function consisted of a simple empirical scoring function and a pharmacophore-based scoring function to reduce the number of false positives. The energy function can be dissected into the following terms:

$$
\mathrm{E}_{\text {tot }}=\mathrm{E}_{\text {bind }}+\mathrm{E}_{\text {pharma }}+\mathrm{E}_{\text {ligpre }}(1)
$$

where $E_{b i n d}$ is the empirical binding energy used during the molecular docking; Epharma is the energy of binding-site pharmacophores; $\mathrm{E}_{\text {ligpre }}$ is a penalty value if the ligand unsatisfied the ligand preferences. $E_{\text {pharma }}$ and $E_{\text {ligpre }}$ were used to improve the number of true positives by discriminating active compounds from hundreds of thousands of non-active compounds. The empirical binding energy $\left(E_{b i n d}\right)$ is given as:

$$
E_{\text {bind }}=E_{\text {inter }}+E_{\text {intra }}+E_{\text {penal }}(2)
$$

where $E_{\text {inter }}$ and $E_{\text {intra }}$ are the intermolecular and intramolecular energy, respectively, $E_{\text {penal }}$ is a large penalty value if the ligand is out of range of the search box. In this paper, $E_{\text {penal }}$ is set to 1000 . For screening: The population size was $=200$, generations $=70$, number of solutions $=3$. Fitness is the total energy of a predicted pose in the binding site. The empirical scoring function of iGEMDOCK is estimates as:

\section{Fitness $=v d W+\mathrm{H}_{\text {bond }}+$ Elec. (3)}

Here, the $v d W$ term is van der Waal energy. $\mathrm{H}_{\text {bond }}$ and Elect terms are hydrogen bonding energy and electro statistic energy, respectively. Screenshots of the ligand-amino acid residue interactions created by Chimera software [12].

Density Functional Theory Studies

Geometric optimization calculations were performed in accordance with DFT method using ORCA software $[13,14]$. Frequency calculations were performed to obtain thermodynamic properties and to verify that each optimization achieved an energy minimum. The quantum chemical descriptors extracted directly from the ORCA output file were total energy, Mulliken atomic charges, electronic density, dipole moment, Mayer population analysis, the energy of the highest occupied molecular orbital (HOMO), and the energy of the lowest unoccupied molecular orbital (LUMO) $[15,16]$. The molecular orbitals and the optimized structures taken by AVOGADRO software [17]. Detailed calculated values can be found in tables in supplementary material. 
The pharmacological profile of the best scoring inhibitors was tested by Toxtree software [18,19], using Cramer rules and Cytochrome P450 metabolism prediction, taking information on pKa values, logP values, solubility, refractivity and estimated toxicity of the molecules.

\section{Results}

SARS-Cov-2 RNA- dependent polymerase binding studies

The 14 drug compounds tested on nsp12 protein via molecular docking studies. In Fig. 1 we can see the crystal structure of the protein together with three drug molecules (chloroquine, remdesivir and saquinavir). SARS-Cov-2 RNA depended polymerase consisted by four chains A, B, C and D. All the tested compounds interacted on $A$ chain but in different sides of the protein. For example, chloroquine seems to prefer the outer structure of the protein, while remdesivir binds in the inner pocket of the protein. At the same figure we can also see the amino acid residues of the A chain that interacting with the three molecules. Remdesivir, interacts via hydrogen bonding with TRP-617, TYR-619, ASP 760, ASP 761, amino acids while interacts with van der Waals forces with the amino acids TYR 455, ARG 553, ASP 618, TYR 619, LYS 621 and ASP 760. Hydrogen bonding interactions are considered hydrophilic interactions while van der Waals forces are considered hydrophobic interactions. Saquinavir interacts with the protein with only two hydrogen bonds with SER 318 and PHE 321. Regarding van der Waals forces, Saquinavir interacts with TYR 265, PHE 321, PRO322, PRO 323, ARG 349 and PRO 461. The amino acids residue is on less than 5 Angstrom distance of the molecules. We are explaining the interactions of Remdesivir and Saquinavir, because they gave the lowest energy values in molecular interactions with the protein, which means that these molecules can form stable complexes with the specific protein and have better binding affinity. Our docking results agree with the predicted pharmacological properties that we are going to see later in this article. Remdesivir is more hydrophilic than hydrophobic molecule while Saquinavir have less solubility in polar environments. The data of the amino acid residues energies of the rest 12 molecules can be found in a table in supplementary material. Additionally, in Table 1 we can find the binding scores (binding energies in $\mathrm{kcal} / \mathrm{mol}$ ) of the 14 drugs that presented in this study. In Table 2 we can observe how this energy distributes in hydrogen bonds, van der Waals forces and electrostatic interactions. Interestingly only Benidipine and Famotidine interacting electrostatically with this protein and the structural activity of molecules that interact electrostatically with the specific protein could be evaluated in another study. Except of the binding energies of the molecules, in Table 1 we can find information on the current uses of the drugs and their current status regarding Covid-19 disease. It seems that our findings are valid as Remdesivir continues with Phase (III) clinical trials. On the other hand, to the best of our knowledge Saquinavir is not participating in any clinical trial and our opinion based on our findings is that it is a promising candidate.

Remdesivir and Saquinavir Computational Studies 
Based on the molecular docking results, we wanted to study further the structures of Remdesivir and Saquinavir in order to try to find a correlation between structure and binding activity for these two molecules. For that reason, we employ computational chemistry studies. DFT reveals the values of the energy difference between HOMO and LUMO as well as the highest occupied molecular orbital (EHOMO) and lowest unoccupied molecular orbital (ELUMO) energies which plays a very important role in stability and reactivity of molecules. The EHOMO energies of molecules show the molecule's ability to give electrons. On the other hand, ELUMO characterizes the ability of the compound to accept electrons. The energy gap $\left(\Delta_{\text {gap }}\right.$ of Remdesivir and Saquinavir are close but Remdesivir has a value of 20.903 ev while Saquinavir a value of $18.037 \mathrm{ev}$. The electronic correlation effects play an essential role in the stability of these type of systems, and therefore, it will affect the equilibrium distances and the interaction energies. Electronegativity $(X)$ is a measure of the power of an atom to attract a bonding pair of electrons. According to this theorem is expressed as follows. Based on equation:

$$
x=-\left(\mathrm{E}_{\mathrm{HOMO}}+\mathrm{E}_{\mathrm{LUMO}}\right) / 2(4)
$$

larger $\Delta_{\text {gap }}$ always indicates lower chemical reactivity and higher kinetic stability of the investigated species. The chemical reactivity of molecules is caused by the simultaneous effect of different parameters. So, we can say that Saquinavir is a little bit more chemical reactive and has lower kinetic stability than Remdesivir. In Table 3 we can see the calculated values based on DFT studies. Detailed calculated values can be found in supplementary material.

\section{Toxicity Studies}

The predicted pharmacological properties of these two candidate molecules are depicted in Table 4. Solubility results of the molecules agreed with docking studies saying that Saquinavir is less polar molecule than Remdesivir. The interesting fact here though is that based on Cramer rules both the molecules are categorised as class (III) toxic which means that their side effects should not overpass. Thus, a more detailed evaluation on the toxicity of Remdesivir and Saquinavir should be done. These two drugs are FDA approved for other conditions but nobody could guaranty the interactions and safety use for that disease (Covid-19) without further studies.

\section{Conclusion}

In this study we performed molecular docking studies on SARS-Cov-2 RNA- dependent polymerase using 14 FDA approved drugs -for several other diseases- as ligands. We found that Remdesivir and Saquinavir had the best binding affinity on that protein, so we decided to evaluated these two structures further. Using DFT studies, we concluded that these two molecules are energetically active and stable in these structures causing inhibition through change of the tertiary structure of the protein. Further theoretical studies found that Remdesivir and Saquinavir, despite their potential as SARS-Cov-2 RNA- dependent polymerase inhibitors, are classified as class III toxic substances, leading to a conclusion that they have 
to be evaluated further before their use for this condition. This computational work revealed those results in just few weeks, and saves enormous amount of time and recourses for medicinal chemists.

\section{Declaration}

\section{Credit authorship contribution statement}

Manos C. Vlasiou: Conceptualization, Methodology, Software, Writing - original draft, Supervision. Kyriakos I. loannou: Writing. Kyriaki S. Pafiti: Writing - review \& editing, Validation.

\section{Declaration of competing interest}

The authors declare that they have no known competing financial interests or personal relationships that could have appeared to influence the work reported in this paper.

\section{Acknowledgements}

The authors want to acknowledge the Pharmacy Programme of the University of Nicosia.

\section{References}

1. Lai, C. C.; Shih, T. P.; Ko, W. C.; Tang, H. J.; Hsueh, P. R. Severe Acute Respiratory Syndrome Coronavirus 2 (SARS-CoV-2) and Coronavirus Disease-2019 (COVID-19): The Epidemic and the Challenges. Int. J. Antimicrob. Agents, 2020, 55 (3), 105924. https://doi.org/10.1016/j.ijantimicag.2020.105924.

2. Abd El-Aziz, T. M.; Stockand, J. D. Recent Progress and Challenges in Drug Development against COVID-19 Coronavirus (SARS-CoV-2) - an Update on the Status. Infect. Genet. Evol., 2020, 83 (April), 104327. https://doi.org/10.1016/j.meegid.2020.104327.

3. Manli Wang, Ruiyuan Cao, Leike Zhang, Xinglou Yang, Jia Liu1, Mingyue Xu, Zhengli Shi, Zhihong $\mathrm{Hu}$, Wu Zhong and Gengfu Xiao, Remdesivir and chloroquine effectively inhibit the recently emerged novel coronavirus (2019-nCoV) in vitro Cell Research (2020) 30:269-271; https://doi.org/10.1038/s41422-020-0282-0.

4. Lung, ; Lin, Y. S.; Yang, Y. H.; Chou, Y. L.; Shu, L. H.; Cheng, Y. C.; Liu, H. Te; Wu, C. The Potential Chemical Structure of Anti-SARS-CoV-2 RNA-Dependent RNA Polymerase. J. Med. Virol., 2020, 92 (6), 693-697. https://doi.org/10.1002/jmv.25761.

5. Donald C. Hall Jr., Hai-Feng Ji, A search for medications to treat COVID-19 via in silico molecular docking models of the SARS-CoV-2 spike glycoprotein and $3 \mathrm{CL}$ protease, Travel Medicine and Infectious Disease 35 (2020) 
6. Manos C. Vlasiou and Kyriaki S. Pafiti, Screening Possible Drug Molecules for Covid-19. The Example of Vanadium (III/IV/V) Complex Molecules with Computational Chemistry and Molecular Docking. DOI: https://doi.org/10.21203/rs.3.rs-34033/v1.

7. Luque, F. J. Frontiers in Computational Chemistry for Drug Discovery. Molecules, 2018, 23 (11). https://doi.org/10.3390/molecules23112872.

8. Hayakawa, D.; Sawada, N.; Watanabe, Y.; Gouda, H. A Molecular Interaction Field Describing Nonconventional Intermolecular Interactions and Its Application to Protein-Ligand Interaction Prediction. J. Mol. Graph. Model., 2020, 96, 107515. https://doi.org/10.1016/j.jmgm.2019.107515

9. Wakui, N.; Yoshino, R.; Yasuo, N.; Ohue, M.; Sekijima, M. Exploring the Selectivity of Inhibitor Complexes with Bcl-2 and Bcl-XL: A Molecular Dynamics Simulation Approach. J. Mol. Graph. Model., 2018, 79, 166-174. https://doi.org/10.1016/j.jmgm.2017.11.011.

10. Ercan, S.; Şenses, Y. Design and Molecular Docking Studies of New Inhibitor Candidates for EBNA1 DNA Binding Site: A Computational Study. Mol. Simul., 2020, 46 (4), 332-339. https://doi.org/10.1080/08927022.2019.1709638.

11. -M. Yang* and C.-C. Chen, "GEMDOCK: A generic evolutionary method for molecular docking," Proteins: Structure, Function, and Bioinformatics, vol. 55, pp. 288-304, 2004.

12. UCSF Chimera-a visualization system for exploratory research and analysis. Pettersen EF, Goddard TD, Huang CC, Couch GS, Greenblatt DM, Meng EC, Ferrin TE. J Comput Chem. 2004 Oct;25(13):1605-12.

13. Neese, F. (2012) The ORCA program system, Wiley Interdiscip. Rev.: Comput. Mol. Sci., 2, 73-78.

14. Neese, F. (2017) Software update: the ORCA program system, version 4.0, Wiley Interdiscip. Rev.: Comput. Mol. Sci., 8 ,

15. Rocha, J. A.; Rego, N. C. S.; Carvalho, B. T. S.; Silva, F. I.; Sousa, J. A.; Ramos, R. M.; Passos, N. G.; De Moraes, J.; Leite, J. R. S. A.; Lima, F. C. A. Computational Quantum Chemistry, Molecular Docking, and ADMET Predictions of Imidazole Alkaloids of Pilocarpus Microphyllus with Schistosomicidal Properties. PLoS One, 2018, 13 (6), 1-23. https://doi.org/10.1371/journal.pone.0198476.

16. Çakmak, E.; Özbakır Işın, D. A Theoretical Evaluation on Free Radical Scavenging Activity of 3Styrylchromone Derivatives: The DFT Study. J. Mol. Model., 2020, 26 (5). https://doi.org/10.1007/s00894-020-04368-7.

17. Marcus D Hanwell, Donald E Curtis, David C Lonie, Tim Vandermeersch, Eva Zurek and Geoffrey R Hutchison; "Avogadro: An advanced semantic chemical editor, visualization, and analysis platform" Journal of Cheminformatics 2012, 4:17.

18. Patlewicz G. Jeliazkova N, Safford RJ Worth AP, Aleksiev B (2008). An evaluation of the implemenation of the Cramer Classification scheme in Toxtree Software. SAR QSAR, Environ Res: 19 (5-6): 495-524.

19. Patrik Rydberg, David E. Gloriam, Jed Zavetzki, Curd Breneman, Lars Olsen, SMARTCyp: A 2D method for the prediction of Cytochrome P450-Mediated Drug Metabolism, ACS Med. Chem. Lett., 2010, 1 (3), p.p 96-100. 


\section{Tables}

Table 1: Representation of the 14 FDA approved drugs with their use, current state on covid-19 clinical trials and calculated binding affinities on SARS-CoV-2 RNA depended Polymerase.

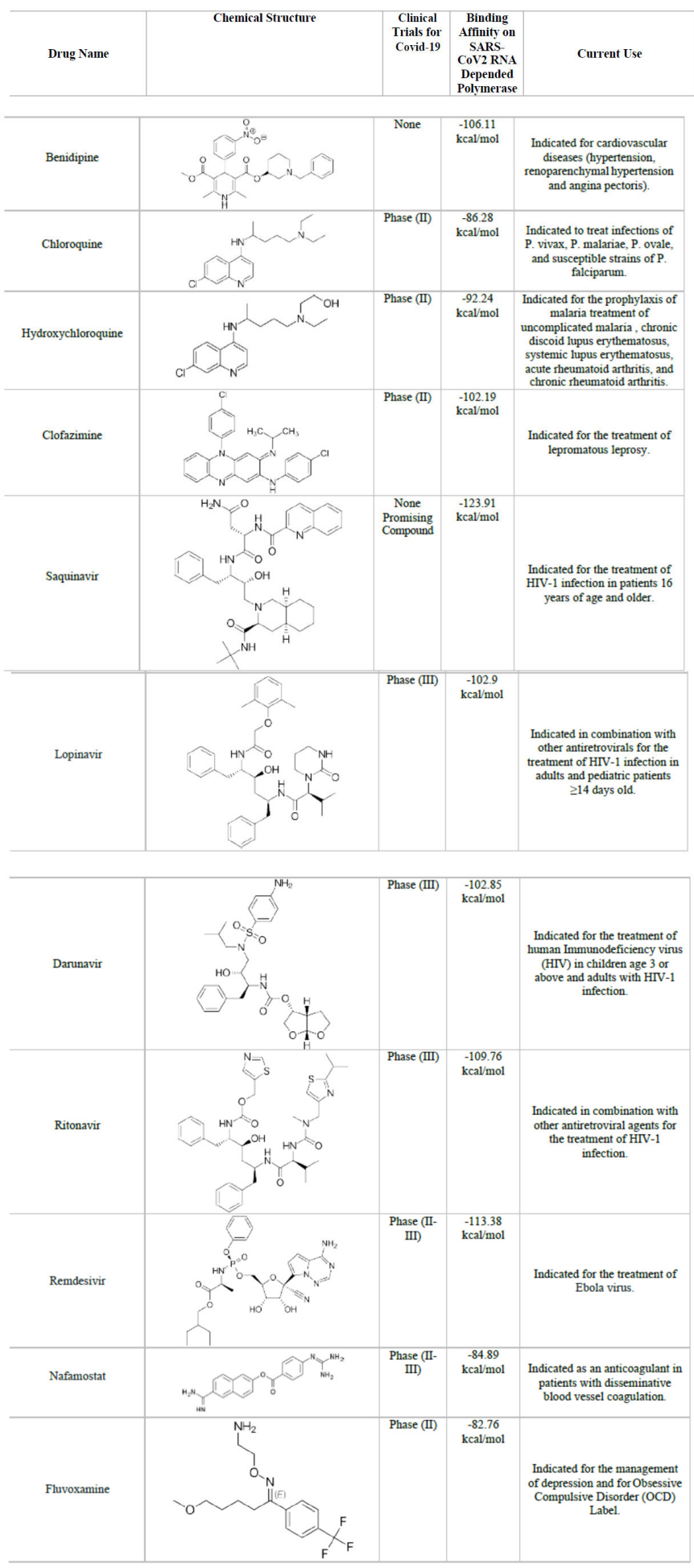


Table 2: Energy distribution of the studied molecules between van der Waals forces, hydrogen bonding and electrostatic interactions.

\begin{tabular}{cccc}
\hline Ligand & VDW & $\mathbf{H}_{\text {bond }}$ & Elec \\
\hline Bemidipine & -85.39 & -20.27 & -0.45 \\
Chloroquine & -82.78 & -3.5 & 0 \\
Clofazimine & -94.92 & -7.27 & 0 \\
Hydroxychloroquine & -87.9 & -4.35 & 0 \\
Saquinavir & -117.56 & -6.36 & 0 \\
Lopinavir & -94.85 & -8.06 & 0 \\
Darunavir & -87.32 & -15.53 & 0 \\
Ritonavir & -95.27 & -14.49 & 0 \\
Remdesivir & -81.98 & -31.4 & 0 \\
Nafamostat & -63.59 & -21 & 0 \\
Fluvoxamine & -79.26 & -3.5 & 0 \\
Famvir & -49.89 & -35.51 & 0 \\
Famotidine & -44.31 & -52.76 & -5.06 \\
Camostat & -69.08 & -24.76 & 0 \\
\hline
\end{tabular}

Table 3: Calculated DFT values of structure details of the two best candidate drugs

\begin{tabular}{ccc}
\hline Calculated Values & Remdesivir & Saquinavir \\
\hline Stretch & 3.6608 & 4.1557 \\
Bend & 27.8165 & 23.3364 \\
Stretch-Bend & -0.1467 & 1.0183 \\
Torsion & 24.7936 & 15.9850 \\
Non-1,4 VDW & -18.3046 & -26.5408 \\
1,4 VDW & 33.4340 & 36.4395 \\
Dipole-Dipole & -1.0265 & -28.1603 \\
Total Energy & $70.2271 \mathrm{kcal} / \mathrm{mol}$ & $26.2339 \mathrm{kcal} / \mathrm{mol}$ \\
\hline
\end{tabular}

Table 4: Pharmacological properties of the two best inhibitor candidates

\begin{tabular}{ccc}
\hline Pharmacological Property & Remdesivir & Saquinavir \\
\hline Log P & 2.20 & 4.04 \\
Log S & -3.25 & -5.43 \\
Solubility & $0.339 \mathrm{~g} / 1$ & $0.0024 \mathrm{~g} / 1$ \\
pKa & 12.14 & 14.24 \\
Refractivity & 161.808 & 186.673 \\
Toxicity (Cramer Rules) & Class (III) & Class (III) \\
\hline
\end{tabular}




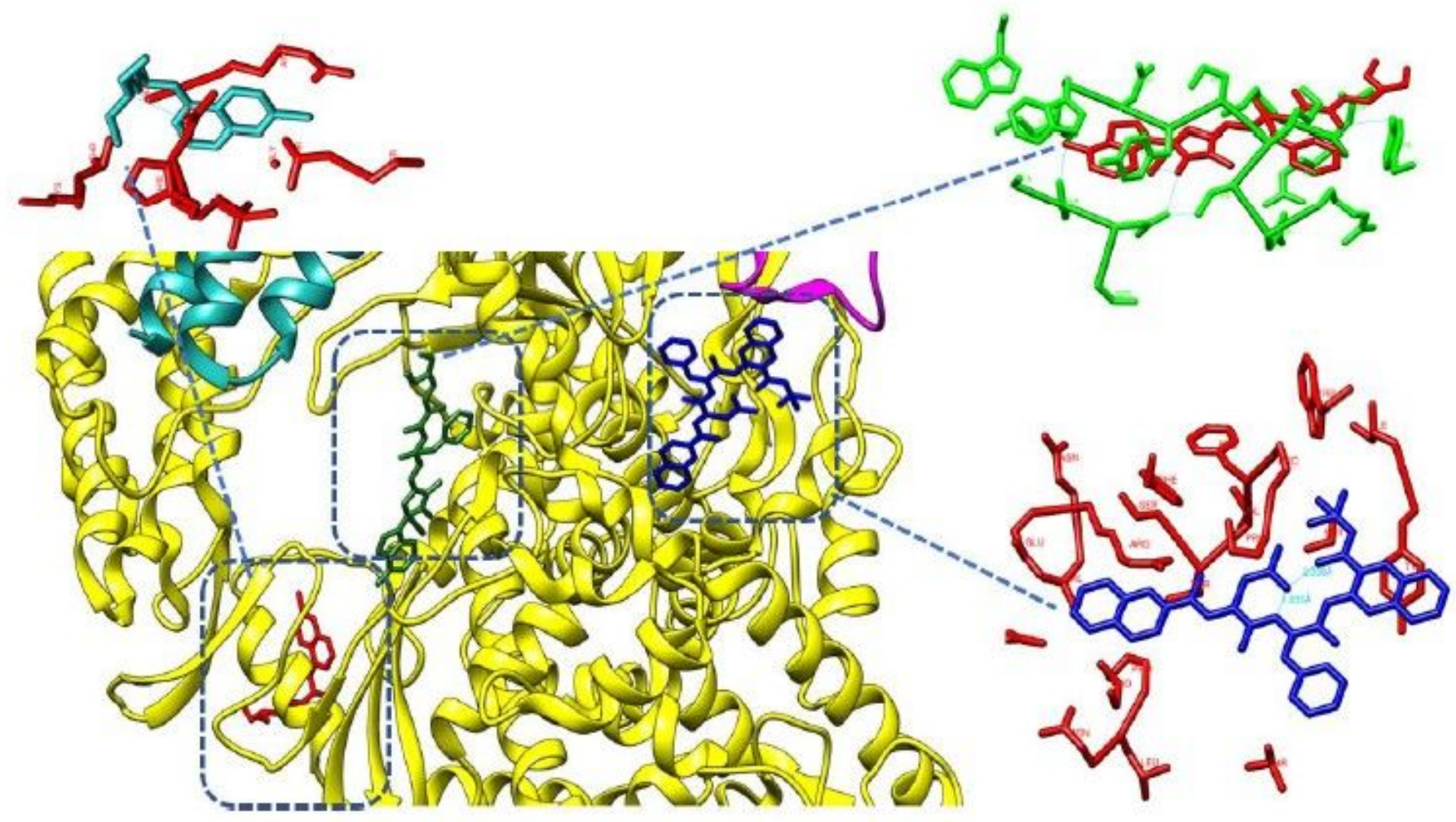

Figure 1

Screenshots of SARS-CoV-2 RNA depended Polymerase crystal structure alongside with the interaction profile of chloroquine (red colour), remdesivir (green colour) and saquinavir (blue colour). The inhibitors can be seen interacting with amino acid residues of the protein as well. 